\title{
Los Estilos De Aprendizaje En Estudiantes De Bachillerato De Una Unidadeducativa Ecuatoriana. Un Estudio De Caso
}

\author{
Learning Styles For Baccalaureate Students In Ecuadorian \\ Schools. A Case Of Study
}

Marcelo Mantilla Falcón

Doctor en Ciencias de la Educación, Magíster en Docencia Universitaria y Administración Educativa. Magister en Ciencias de la Educación. Docente en la Facultad de Contabilidad y Auditoría de la Universidad Técnica de Ambato. luismmantilla@uta.edu.ec

\section{Resumen}

El presente estudio busca determinar la relación existente entre los estilos de aprendizaje con el uso del test CHAEA, aplicados a estudiantes de tercer año de bachillerato de una unidad educativa del Ecuador. La investigación realiza un análisis multifactorial y multivariado con los 4 elementos que mide el test de estilos de aprendizaje. Es un estudio empírico, transversal, no experimental, de carácter cuantitativo y descriptivo, la muestra incluyó 231 estudiantes de los dos géneros. Esta investigación pretende explicar el vacío de conocimientos en esta realidad educativa para el contexto local ecuatoriano. Los resultados más evidentes demuestran que los estilos de aprendizaje están fuertemente correlacionados y que hay diferencias significativas entre los bachilleratos.

Palabras clave: estilos de aprendizaje, test, bachillerato, educación, pedagogía.

\begin{abstract}
This study aims to determine the relationship between learning styles using CHAEA test applied to third-year students of Baccalaureate of an educational unit of Ecuador. The research makes a multifactorial and multivariate analysis with the 4 elements test that measures learning styles. It is an empirical, cross-sectional, not experimental, quantitative and descriptive approach; the sample included 231 students of both genders. This research aims to explain the knowledge gap in this educational reality for the Ecuadorian local context. The most obvious results show that learning styles are strongly correlated and that there are significant differences between high schools.
\end{abstract}

Keywords: learning styles, test, baccalaureate, education, teaching. 


\section{Introducción.}

Los estilos de aprendizaje ya tienen algunas décadas de ser estudiados y constan en la literatura académica pedagógica con múltiples conceptos y definiciones desarrolladas para el efecto, sin embargo, Keefe (1982) citado por Gallego y Alonso (2010), proponen un concepto más ajustado a los requerimientos de este estudio y que Gallego y Alonso se apropian para sus análisis en los siguientes términos: "son rasgos cognitivos, afectivos y fisiológicos, que sirve como indicadores relativamente estables, de cómo perciben los dicentes, interaccionan y responden a sus ambientes de aprendizaje".

Autores como Alvarado (2015) analizan la relación y la influencia de los Estilos de Aprendizaje (EA) y el diseño de mapas mentales con el fin de identificar cuáles estilos de aprendizaje se benefician con el uso de este tipo de organizadores gráficos. El autor verifica que los estudiantes con estilos Auditivo y Divergente fueron los que presentaron el mejor desempeño al diseñar los mapas mentales. Sin embargo, a partir del test de Kolb se observa más diversidad en los estilos para procesar, por lo que es necesario tener en cuenta las características de los procesos cognitivos de acuerdo con las edades en los grados.
Los estudios sobre estilos de aprendizaje se hacen en todos los niveles educativos, tal es el caso realizado por Román, Díaz y Leyva (2015) en estudiantes que se formaron en la carrera de Enfermería en un estado mexicano, estos son algunos de los resultados: el estilo de ingreso fue reflexivo, $39.3 \%$, seguido del pragmático; al egreso $22.2 \%$ lo conservó y $17.1 \%$ lo modificó; la coincidencia entre estilo de ingreso y egreso fue mínima.

Otro estudio importante a nivel universitario es el de Villalva (2015) en el que se evaluó el perfil de estilos de aprendizaje a través de la aplicación del CHAEA sobre una muestra de 109 alumnos universitarios, de los cuales 72 eran ingresantes y 37 próximos a egresar, de las carreras del Profesorado en Biología y la Licenciatura en Biodiversidad de la Facultad de Humanidades y Ciencias de la Universidad Nacional del Litoral. Se consideró el efecto de las variables independientes: curso, edad, género, escuela de procedencia, lugar de residencia y carrera sobre los estilos de aprendizaje, de manera individual y conjunta. Se registran diferencias significativas entre los grupos con respecto a los estilos Activo y Pragmático, estando estos más desarrollados en los alumnos ingresantes. Los alumnos con 21 años o menos y las mujeres prefieren el estilo Reflexivo. 
Otro de los instrumentos para evaluar los Estilos de Aprendizaje es el de Felder y Silverman, el cual clasifica los estilos de aprendizaje a partir de cinco dimensiones, las cuales están relacionadas con la información que perciben los estudiantes, la modalidad sensorial que les permita recibirla mejor, la forma como se organiza esa información, como la procesa y como progresa en su aprendizaje, bajo estas consideraciones Tocci (2015) evidencia estos resultados en su investigación con estudiantes de ingeniería de la Universidad Nacional de La Plata, Argentina: los cuestionarios fueron respondidos por un total de 185 alumnos, de los cuales 43 son de la especialidad Química, 23 de Electromecánica, 51 Electrónica, 19 de Mecánica, 13 de Aeronáutica y 36 de Electricista. No hubo diferencias en cuanto a los perfiles de estilos según la especialidad elegida, por eso se hizo una generalización de alumnos de Ingeniería sin tener en cuenta que rama seguían. De acuerdo a los resultados obtenidos se ve que hay un equilibrio entre activos y reflexivos, los primeros tienden a retener y comprender mejor la nueva información cuando hacen algo activo con ella por ejemplo mediante un debate, o explicándosela a un compañero o trabajando en grupo, los segundos en cambio tienden a retener la información pensando y reflexionando sobre ella.

En el mismo estudio, el autor ve que hay un equilibrio entre activos y reflexivos, los primeros tienden a retener y comprender mejor la nueva información cuando hacen algo activo con ella por ejemplo mediante un debate, o explicándosela a un compañero o trabajando en grupo, los segundos en cambio tienden a retener la información pensando y reflexionando sobre ella. Las evidencias llevan a pensar que aquellos que son activos organizaran la información de forma inductiva a partir de las observaciones en el campo, en cambio aquellos que son reflexivos seguramente lo harán en forma deductiva.

Los autores Páez y Malagón (2015) se propusieron verificar el impacto de los Estilos de Aprendizaje desde la óptica de la educación diferenciada, considerando el género y los estilos cognitivos, en estudiantes de noveno grado de una institución de Bogotá-Colombia. Se diseñó un estudio transversal, desde un enfoque cuantitativo y alcance descriptivo, aplicando el cuestionario Honey-Alonso para Estilos de Aprendizaje (CHAEA) y el test de figuras enmascaradas (EFT) para estilos cognitivos, a 38 hombres y 58 mujeres del grado noveno.

Los resultados, después de efectuar pruebas $t$ de student y coeficientes de correlación, muestran diferencias significativas por género en la relación entre los estilos de aprendizaje, cognitivos y el logro académico, siendo privilegiadas las mujeres por este modelo educativo. 
Considerando el modelo del Kolb para evaluar o determinar los estilos de Aprendizaje, el estudio de los autores Sánchez, Donat, Fornons y Vaqué (2015) con estudiantes universitarios y profesionales de la salud en una institución catalana, se evidenciaron resultados importantes: De los 288 individuos que constituyeron la muestra, 22 eran fisioterapeutas voluntarios $\quad(7,6 \%), \quad 32$ profesores universitarios (11,1\%) y 234 estudiantes de Ciencias de la Salud (81,3\%), distribuidos por estudios matriculados y curso académico. Participaron 175 mujeres $(60,8 \%)$ y 113 hombres (39,2\%), con edades comprendidas entre los 15 y 53 años y una media de edad de 24,02 (DE=7,577), mayoritariamente distribuidos en un rango de 18 a 23 años (67\%). Se encontró que están presentes los cuatro Estilos de Aprendizaje (EA) incluidos en el modelo. El EA mayoritariamente encontrado fue el estilo Divergente (69,79\%), seguido del Adaptador $(21,18 \%)$, en menor grado el Asimilador $(6,25 \%)$ y por último el estilo Convergente, hallado en 8 estudiantes $(2,77 \%)$ y ausente entre los profesionales de Ciencias de la Salud. En todos los grupos (estudiantes, profesores y fisioterapeutas cooperantes) predominó el estilo Divergente. Los estudiantes y profesores estudiados tienen un EA preferentemente divergente, que se manifiesta de forma mayoritaria en el colectivo.
Hay estudios donde se contrastan los Estilos de Aprendizaje con la destreza de adquisición de una segunda lengua, en este caso el inglés. El estudio tuvo como objetivo identificar la tendencia de estilos de aprendizaje utilizados por los estudiantes del Centro Boliviano Americano de la ciudad de SucreBolivia, que estudian el inglés como segunda lengua. Adicionalmente se analizó también la relación que existe entre el estilo de aprendizaje utilizado y el género, edad, grado de instrucción y el tiempo en el que las personas estudian el idioma. Para ello seleccionó una muestra representativa de 173 estudiantes de una población de 1273 y se aplicó el Cuestionario de Honey-Alonso para Estilos de Aprendizaje CHAEA que los clasifica en: activo, reflexivo, teórico y pragmático. Los resultados muestran una leve tendencia hacia el estilo reflexivo, pero en general se detectó un dominio homogéneo de los cuatro estilos. También se encontró una leve correlación significativa entre el género y el estilo pragmático, entre la edad y el estilo reflexivo; y entre el grado de instrucción y los estilos de aprendizaje activo y reflexivo. Se concluye que es necesario realizar no sólo un análisis inicial sino un seguimiento periódico al proceso de evaluación del aprendizaje, ya sea a través de herramientas disponibles o adaptadas al contexto (Guillen, 2015).

Los estilos de Aprendizaje se vinculan con el manejo de las TIC en el estudio desarrollado por Hernández, García, 
Fernández y Medina (2012) se hace un estudio preliminar para conocer la forma en que los docentes utilizan las TIC en sus cursos, en especial el uso de hardware, software y plataformas educativas. Sus resultados son: Los profesores tienen mayores preferencias en cuanto a los Estilos de Aprendizaje Reflexivo y Teórico, Además, tienen, en promedio, bajos valores en los estilos Pragmático y Activo. Los académicos del CP regularmente son metódicos, lógicos, objetivos, críticos y estructurados; prefieren ser ponderados, concienzudos, receptivos, analíticos y exhaustivos. Los profesores con preferencia alta en los Estilos Activo y Pragmático hacen mayor uso de herramientas colaborativas y redes sociales. Los docentes con preferencias altas en los Estilos Reflexivo y Pragmático hacen uso de plataformas educativas.

Es tan decisor lo que asevera la Unión Europea (2007) al exponer que "El aprendizaje permanente se ha convertido en una necesidad para todos los ciudadanos. Necesitamos mejorar nuestras aptitudes y competencias a lo largo de toda nuestra vida, no solo para realizarnos personalmente y ser capaces de participar activamente en la sociedad en que vivimos, sino también para poder tener éxito en un mundo laboral en constante evolución".

\section{Metodología}

Se trata de una investigación con enfoque cuantitativo de carácter descriptivo, transversal, no probabilística y no experimental. Los elementos de observación fueron estudiantes del último año de bachillerato en número de 231, pertenecientes a una institución educativa de nivel secundario ecuatoriana, (Ambato). La participación estudiantil fue el $62,34 \%$ del bachillerato en ciencias y 37,66 del técnico; $48,48 \%$ de género masculino y $51,52 \%$ del femenino. Las edades de los estudiantes oscilaron entre los 16 y 21 años. El test para la toma de datos fue el CHAEA (Cuestionario Honey-Alonso de Estilos de Aprendizaje) (Alonso, Gallego, \& Honey, 1994), mismo que determina los estilos activo, reflexivo, teórico y pragmático.

\section{Resultados}

Tabla 1. Estadística descriptiva de los estilos de aprendizaje global

\begin{tabular}{lllll}
\hline ESTADÍSTICOS & ACTIVO & REFLEXIVO & TEÓRICO & PRAGMÁTICO \\
\hline Media & 12,68 & 12,90 & 12,68 & 13,34 \\
Error típico & 0,18 & 0,20 & 0,18 & 0,19 \\
Mediana & 13 & 13 & 13 & 13 \\
Moda & 14 & 13 & 12 & 13 \\
Desviación estándar & 2,68 & 3,04 & 2,70 & 2,94 \\
Varianza de la muestra & 7,19 & 9,26 & 7,30 & 8,62 \\
Cuenta & 231 & 231 & 231 & 231 \\
\hline
\end{tabular}

Fuente: Encuesta a los estudiantes 
De acuerdo con el Test CHAEA, se evidencia que el Estilo Pragmático es el que alcanzó un promedio más elevado con 13,3 puntos, considerando que los cuatro estilos pueden tener como máximo 20 puntos. Le sigue en orden de importancia el Estilo Reflexivo. Se igualan en puntos, el Activo y el Teórico.

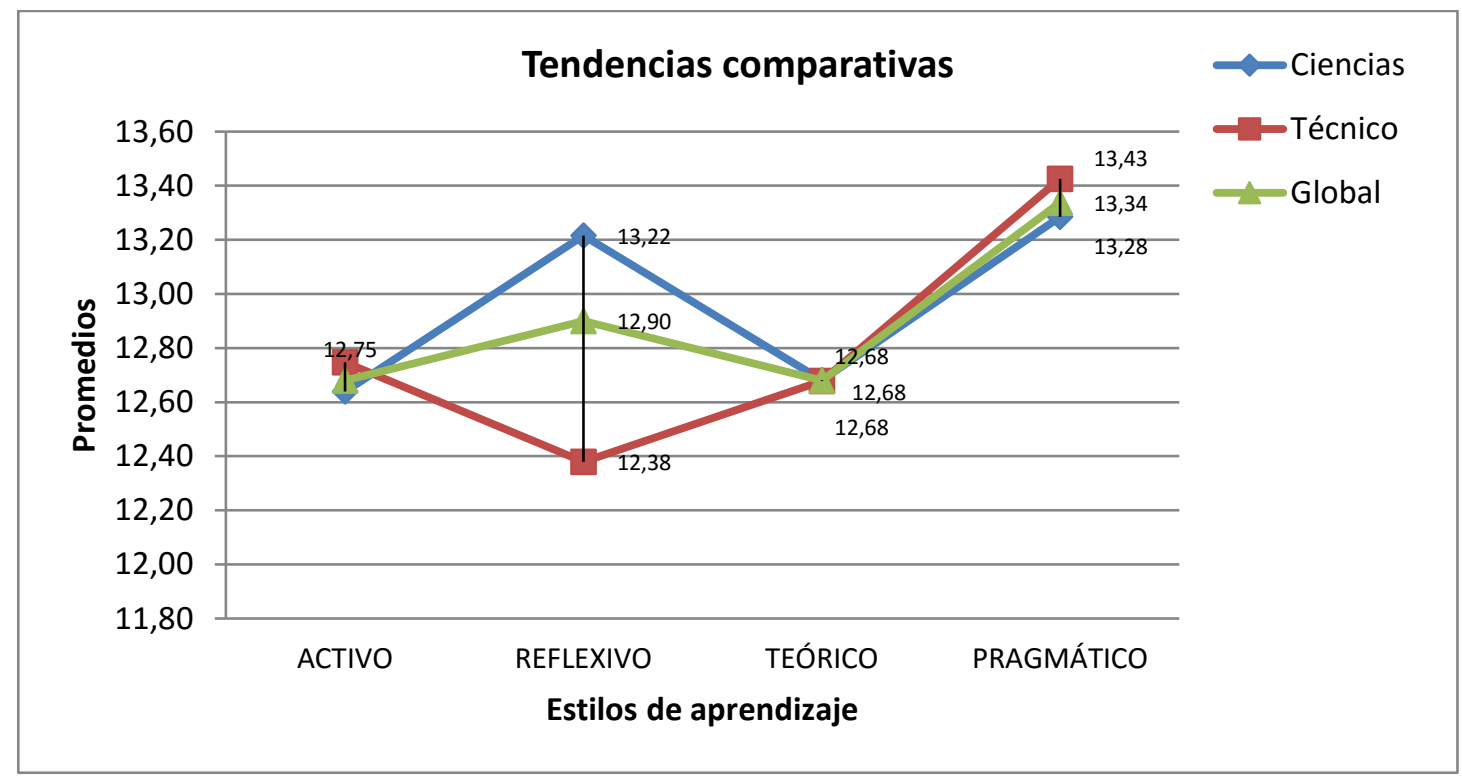

Gráfico 1. Tendencias de los estilos de aprendizaje por bachillerato.

Las tendencias comparativas entre los dos tipos de bachillerato en función de sus estilos de aprendizaje, tienen tres estilos coincidentes: Activo, Teórico y Pragmático, el punto disidente radica en el estilo Reflexivo con una marcada diferencia. La tendencia de los estilos en función del tipo de bachillerato tiene la misma orientación de los datos consolidados.

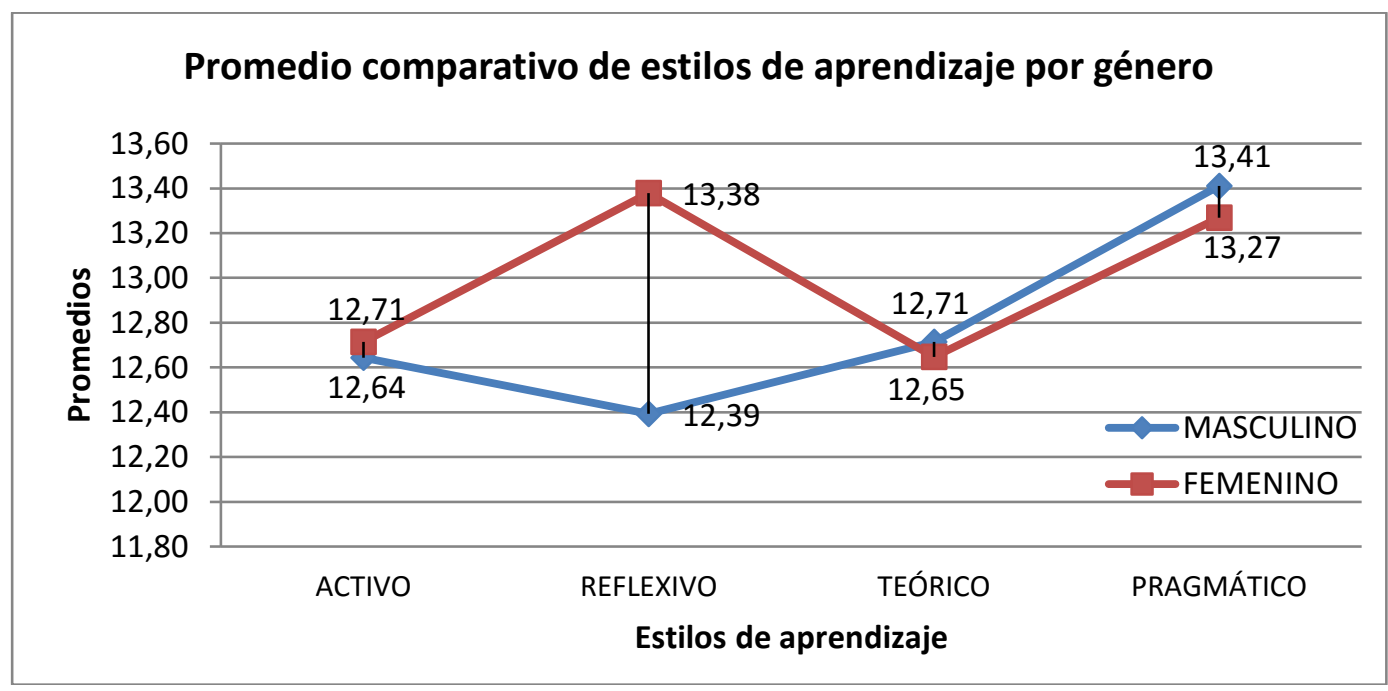

Gráfico 2. Tendencias de los estilos de aprendizaje por género.

26 FALCÓN, M.M.: Los Estilos De Aprendizaje En Estudiantes De Bachillerato De Una Unidadeducativa Ecuatoriana. Un Estudio De Caso. 
El estilo diferenciador entre géneros es el Reflexivo donde se evidencia una fuerte preferencia por el género femenino, los otros tres estilos presentan la misma orientación con diferencias de puntuación muy reducida y poco significativa.
Alonso y otros (1994, pág. 114) proponen las puntuaciones de cada uno de los Estilos de Aprendizaje por cada una de las preferencias como se muestra en la tabla "Baremo General de Preferencia en Estilos de Aprendizaje".

Tabla 2. Baremos de los estilos de aprendizaje

\begin{tabular}{|c|c|c|c|c|c|}
\hline PORCENTAJES & $10 \%$ & $20 \%$ & $40 \%$ & $20 \%$ & $10 \%$ \\
\hline \multirow{2}{*}{ ESCALAS } & \multicolumn{5}{|c|}{ Preferencias } \\
\hline & Muy Baja & Baja & Moderada & Alta & Muy Alta \\
\hline T. APRENDIZAJES & MB & B & M & A & MA \\
\hline Activo & $0-6$ & $7-8$ & $9-12$ & $13-14$ & $15-20$ \\
\hline Reflexivo & $0-10$ & $11-13$ & $14-17$ & $18-19$ & 20 \\
\hline Teórico & $0-6$ & $7-9$ & $10-13$ & $14-15$ & $16-20$ \\
\hline Pragmático & $0-8$ & $9-10$ & $11-13$ & $14-15$ & $16-20$ \\
\hline
\end{tabular}

Fuente: Alonso, Gallego y Honey (1999).

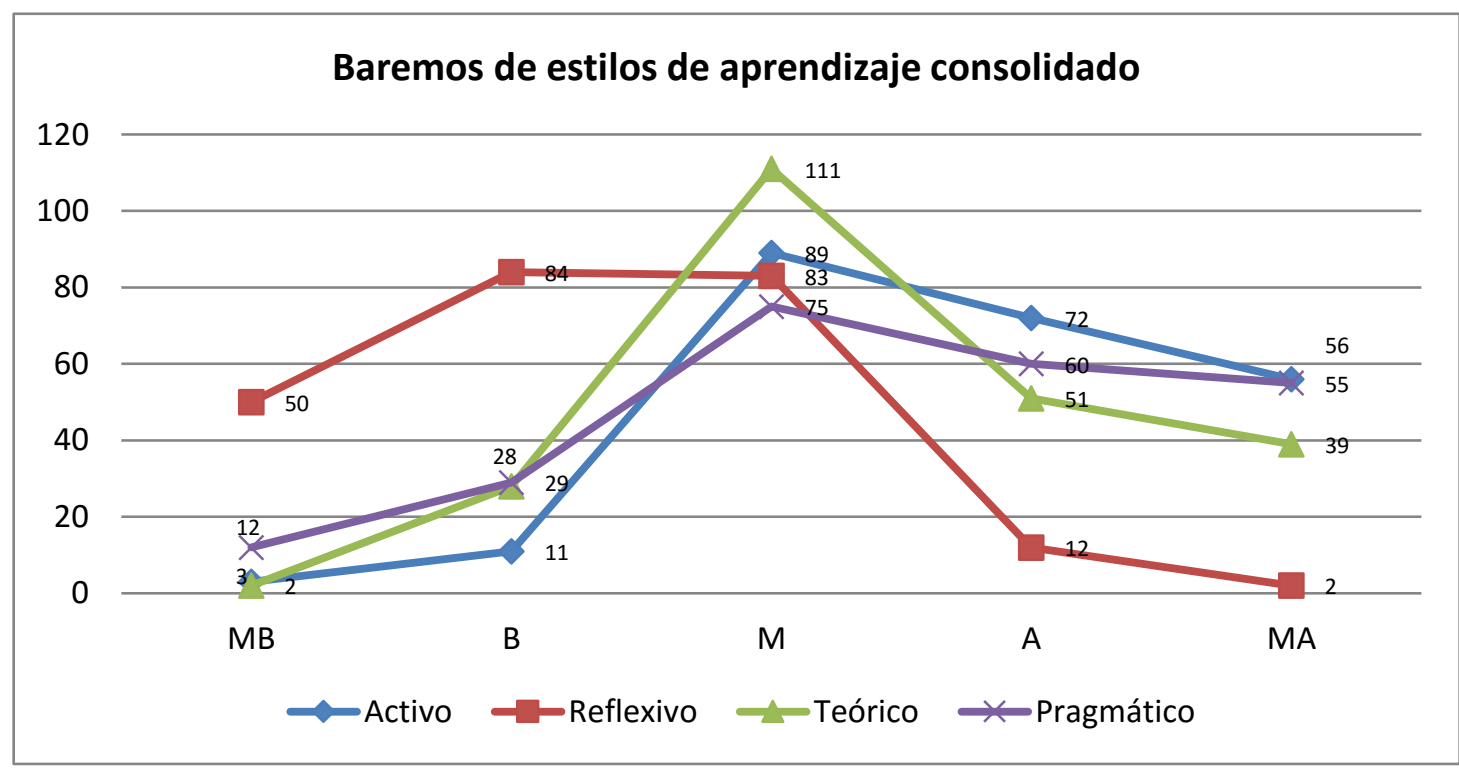

Gráfico 3. Baremos de los estilos de aprendizaje consolidado.

Los datos consolidados demuestran una distribución uniforme entre los estilos Activo, Teórico y Pragmático, mientras que el Estilo Reflexivo marca la diferencia en el baremo "Muy Baja, MB" y "Baja, B". Analizando mediante la prueba de Ji cuadrado se obtiene esta información: $\mathrm{X}^{2}{ }_{\mathrm{c}}(271,00) ; \mathrm{gl}=12 ; p>$ 0,000 , con $\alpha$ de 0,05 . Estos resultados quieren decir que hay diferencia altamente significativa entre los estilos de aprendizaje y la distribución de baremos en el grupo de estudio analizado. 
Tabla 3. Correlación interna de Estilos de Aprendizaje

\begin{tabular}{|c|c|c|c|c|}
\hline ESTILOS & Activo & Reflexivo & Teórico & Pragmático \\
\hline Activo & 1 & & & \\
\hline Reflexivo &, $160^{*}$ & 1 & & \\
\hline Teórico &, $157^{*}$ &, $401^{* *}$ & 1 & \\
\hline Pragmático &, $229^{* *}$ &, $355^{* *}$ &, $389^{* *}$ & 1 \\
\hline
\end{tabular}

La correlación evidencia que los estilos mejor ajustados es el Teórico versus el Reflexivo con un $\mathrm{r}$ de 0,401, cuyas características, a más de las ya expuestas anteriormente se detallan a continuación como bien lo define Curet (2011) .

\section{Discusión}

Estos estilos, de acuerdo con el modelo teórico de Honey y Mumford, fueron modificados en su contenido por Alonso, Gallego y Honey (1994), quienes los redefinen como:

a) Activo.- Corresponde a las personas que se caracterizan por ser animadoras, improvisadoras, descubridoras, espontáneas y arriesgadas. Están interesadas en vivir las experiencias y ser cambiantes. Este estilo tiene alta homogeneidad en los dos bachilleratos por tanto, sus valores coinciden aun con el promedio general del grupo de estudio.

b) Reflexivo.- Incluye a las personas que son ponderadas, receptivas, analíticas $y$ exhaustivas. Son observadoras, pacientes, detallistas, investigadoras y asimiladoras. La disparidad entre bachilleratos es muy importante, se oponen diametralmente, en el técnico tiene una puntuación promedio de 12 , 38, mientras que en bachillerato en ciencias alcanza un promedio de 13,22. Son más reflexivos los estudiantes de Ciencias que los Técnicos.

c) Teórico.- Caracteriza a las personas que son metódicas, lógicas, objetivas, críticas y estructuradas; son disciplinadas, ordenadas, buscadoras de hipótesis y teorías, además de exploradoras. Es un estilo que tiene una coincidencia perfecta $(12,68)$ en los dos bachilleratos y también se nivela con el promedio global del grupo de estudio.

d) Pragmático.- Incluye a las personas experimentadoras, prácticas, eficaces y realistas; se caracterizan por ser rápidas, organizadoras, estar seguras de sí mismas, de solucionar problemas y de planificar sus acciones. Los estudiantes del bachillerato técnico alcanzaron el mayor promedio con 13,43, sin embargo, los estudiantes de ciencias no distan mucho de este estilo, su promedio es de 13,28. 
Mediante un ANOVA se determina que si hay diferencia estadística significativa entre los Estilos de Aprendizaje en el tercer año de bachillerato de la Unidad Educativa Rumiñahui con un $\alpha$ de 0,05 y un $p<0,042 .\left(\mathrm{F}_{2,615}=\right.$ 2,746).

\section{Conclusiones}

-Se determina la relación directamente proporcional entre los estilos de aprendizaje, siendo los Estilos: Reflexivo, Teórico y Pragmático, los más significativos.

-Los estilos de aprendizaje por género tienen alta similitud entre ellos excepto el reflexivo cuya mayor preferencia se da en la mujeres.

-Tomando en consideración los baremos con los que se distribuyen los estilos de aprendizaje de acuerdo al test, existen diferencias altamente significativas entre los participantes del estudio, sin embargo las tendencias son similares. El análisis de varianza también lo confirma.

-El tipo de aprendizaje con mayor presencia en los dos bachilleratos es el pragmático con 13,34 puntos sobre 20. El estilo Reflexivo marca una gran diferencia en los dos tipos de aprendizaje.

\section{Bibliografía}

1. Alonso, C., Gallego, D., \& Honey, P. (1994). Los Estilos de Aprendizaje: Procedimientos de diagnóstico y mejora. Bilbao: Ediciones Mensajero.

Alonso, C., Gallego, D., \& Honey, P. (1999). Los estilos de aprendizaje (Quinta ed.). Bilbao: Mensajero.

Alvarado, L. J. (2015). Estilos de aprendizaje y mapas mentales en estudiantes de secundaria. Revista de Estilos de Aprendiraje, 8(16), 1-24.

Curet, N. (2011). Análisis de los Estilos de Aprendizaje de los estudiantes extranjeros y los estilos de enseñanza de los profesores de tres universidades privadas de Puerto Rico. Tesis inédita. Gurabo: Universidad del Turabo.

Gallego, D. J., \& Alonso, C. M. (2010). Estilos de Aprendizaje. En J. L. García, J. A. Santizo, \& M. A. Jiménez (Edits.), Estilos de Aprendizaje (págs. 24-48). México D.F., México: Colegio de Postgraduados.

Guillen, L. A. (2015). Diagnóstico de los Estilos de Aprendizaje utilizados para la adquisición del inglés como segunda lengua en el Centro Boliviano Americano-Sucre. Revista de Estilos de Aprendizaje, 8(16), 162-177.

Hernández, J., García, J. L., Fernández, Y. M., \& Medina, R. C. (2012). Propuesta de una Memoria de Recursos Didácticos de Gestión vía Web considerando su natuiraleza semántica y los Estilos de Aprendizaje. Revista de Estilos de Aprendizaje, 9(9), 21-46.

Páez, A., \& Malagón, E. (2015). Una mirada desde la educación diferenciada por 
género en el Colegio Gimnasio Vermont, Bogotá-Colombia. Revista de Estilos de Aprendizaje, 8(16), 50-78.

Román, L., Díaz, L., \& Leyva, E. G. (2015). Modificación de los Estilos de Aprendizaje durante la formación profesional, en estudiantes de enfermería. Revista de Estilos de Aprendizaje, 8(16), 25-48.

Sánchez, V., Donat, R., Fornons, D., \& Vqué, C. (2015). Análisis del comportamiento de los Estilos de Aprendizaje en estudiantes universitarios $\mathrm{y}$ profesionales de Ciencias de la Salud. Revista de Estilos de Aprendizaje, 8(16), 137-161.
Tocci, A. M. (2015). Carcaterización de estilos de aprendixzaje en alumnos de ingeniería segun el modelo de Felder y Silverman. Revista de Estilos de Aprendizaje, 8(16), 101-118.

Unión Europea. (2007). Competencias clave para el aprendizaje permanente. Un Marco de Referencia Europeo. Luxemburgo: Oficina de Publicaciones Oficiales de las Comunidades Europeas.

Villalva, A. B. (2015). Estilos de Aprendizaje en alumnos universitarios de profesorado en Biología y Licenciatura en Biodiversidad. Revista de Estilos de Aprendizaje, 8(16), 79-100. 